\title{
A CRITIQUE OF CONTEMPORARY ARTIFICIAL INTELLIGENCE ART: WHO IS EDMOND DE BELAMY?
}

\section{Mikel Arbiza Goenaga}

Sinnergiak Social Innovation Center (Donostia-San Sebastián) Galapagos Studio (Donostia-San Sebastián)

\section{Abstract}

Edmond de Belamy is a 2018 painting made by french collective Obvious, created using a type of Artificial Intelligence algorithms called Generative Adversarial Networks, which was sold at Christie's auction house in New York for $\$ 432,500$. This historic event -the so-called auction of the "first artwork made by an Al" raises 3 interesting questions about authorship, originality, and the arts as a space for scientific inquiry. While some think that the current deployment of Machine Learning algorithms and Artificial Intelligence techniques that we are seeing in the art world today may be seen as the ultimate "Gesamtkunstwerk" or total artwork, other points of view express that not only we need this type of cultural artifacts as a critique of industrialized use of Artificial Intelligence, but also a strict criteria has to be delimited in order to review contemporary art made with Machine Learning techniques.

Keywords: NEUROSCIENCE; ARTIFICIAL INTELLIGENCE; DEEP LEARNING; NEURAL NETWORKS; BLOCKCHAIN; COMPUTER SCIENCE; GANISM; ART; MARKET; CREATIVITY; COMPUTATIONAL AESTHETICS

\footnotetext{
Arbiza Goenaga, Mikel. 2020. "A critique of contemporary artificial intelligence art: Who is Edmond de Belamy?". AusArt 8 (1): 49-64. DOI: $10.1387 /$ ausart.21490
}

\section{AUSART}




\section{ARTIFICIAL INTELLIGENCE: A REVOLUTION IN THE ART MARKET}

\section{1. - WhO IS Edmond de Belamy?}

Edmond de Belamy is a 2018 painting created using a type of Artificial Intelligence algorithms called Generative Adversarial Networks. The work which is part of a series of images titled La Famille de Belamy -made by french collective Obvious- was expected to be sold for $\$ 7,000-\$ 10,000$ at Christie's auction house in New York, however the painting was finally purchased for $\$ 432,500$.

Obvious was founded by 3 young students who are based in Paris: Pierre Fautrel, Hugo Caselles-Dupré and Gauthier Vernier. The main goal of this group of friends, artists, and researchers is to explain and democratize the advances in Artificial Intelligence through art. They started working together in 2017 when they discovered a set of Deep Learning algorithms that generate images called Generative Adversarial Networks (GANs).

Deep Learning is a revolutionary form of Machine Learning made with different layers of Artificial Neural Networks, a set of algorithms modeled loosely after the human brain that are designed to recognize patterns. They interpret sensory data through a kind of machine perception, labeling or clustering raw input. Therefore, Deep Learning is a type of Artificial Intelligence architecture that makes computers learn from experience and understand the world in terms of a hierarchy of concepts by building them out of simpler ones. Since the computer gathers knowledge from the experience of processing different data-sets, there is no need for a human operator to formally specify all of the knowledge needed by the computer.

GANs are a type of framework for the design of Artificial Intelligences which was invented by lan Goodfellow in 2014. The system consist of one network that generates new data after learning from a training set, and another that tries to discriminate between real and fake data. By working together, these networks can produce very realistic synthetic data. According to Yoshua Bengio, one of the world's leading experts on Machine Learning, this approach offers a powerful way for computers to learn from unlabeled data and will help make software that learns without explicit instruction. 
We agree with web-developer Afaan Bilal (2018) that the idea of something that can learn and adapt to changing circumstances and produce information and data relevant in solving real world problems has always been one of the top-most researched areas in the field of Computer Science. Consequently the growing importance of Deep Learning architectures such as Artificial Neural Networks (ANN), used in Generative Adversarial Networks for example, a kind of Computational Connectionist Systems inspired by the biological neural networks that constitute animal brains. This systems learn to do tasks by considering examples without task-specific programming. As neuroscience has shown, all information that our brain processes and stores is done by the way of connections and networks between different neurons and that's the concept on which this Artificial Neural Networks (ANN) are based upon.

This theoretical framework is called Connectionism, which is an approach in the fields of cognitive and computer sciences that hopes to explain mental phenomena using ANN. But while this technology was initially designed to function like biological neural networks, we agree with professor Margaret Boden (2012) that the activity in our brains is far more complex than might be suggested by simply studying artificial neurons. According to Dr. Gaetano Licata the high complexity of the human brain makes it impossible to consider neural networks as good models for human mind, but that doesn't mean they are not good devices for computation in parallel. In contrast Blake A Richards, a professor in University of Toronto, notes that in the next decade discoveries by scientists will put us on the path of new Al which will help us understand our experimental data in neuroscience.

According to programmer and Deep Learning enthusiast Jonathan Hui (2018), Generative Adversarial Networks (GAN) are about creating. Like drawing a portrait or composing a symphony the main focus for GAN is to generate data from scratch. As stated before GANs are designed with of two Artificial Neural Networks, the generator that feeds with data and the discriminator which acts as a critic. Just because a generator alone will just create random noise, conceptually the discriminator provides guidance to the generator on what images to create. By training both networks into a fierce competition to improve themselves, eventually the generator creates images that the discriminator cannot tell the difference and the GAN model eventually converges and produces natural looking images.

This historic event -the so-called auction of the 'first artwork made by an Al' (of course we are omitting the pioneering job of Harold Cohen's robot painter AARON, for example)- raises 3 interesting questions about authorship, originality, and the arts as a space for scientific inquiry: 
1) Authorship: Frédérique Baumgartner, an art historian at Columbia University, says that the portrait of Edmond de Belamy has a light semblance with those paintings made by the old master Rembrandt. In the past, artist like Marcel Duchamp also raised questions about intention and authorship when he emphasized that the artist's idea was the key element in the creation in clear opposition to the classical perspective centered in technique and aesthetics.

This new art created by Generative Adversarial Networks radically flips this idea, not just creating a new thought for an object, but creating an artifact (the Al) capable of doing the thinking and creating for us. Computational systems are not human and so we should accept the creativity they exhibit as legitime creativity, but not as we know it since they will never be exactly the same as the one homo sapiens exhibits.

2) Originality: Google Al engineer François Chollet, creator of an Open Source Neural Network library called Keras, proposed a seductive name for this kind of artworks: GANism ${ }^{1}$. In general, Open Source refers to any program or cultural artifact whose source code or components are made available for use or modification as users or other developers see fit. When Hugo Caselles-Dupré, the tech lead for Obvious and a Machine Learning PhD student in Paris, found artist Robbie Barrat 's's open-source algorithms in code sharing website Github he simply used them to generate the Edmond de Belamy portrait. Does this make the artwork less original?

From sampling in hip-hop to the influence of african-art in Picasso's paintings, the creative world is full of examples of artworks made by recombination.

3) The arts as a space for scientific inquiry: Ahmed Elgammal, professor of Computer Science at Rutgers University and director of the Art and Artificial Intelligence Laboratory, told world-famous auction house Christies, in relation to the artworks created by their Al, that "if you consider the whole process, then what you have is something more like conceptual art than traditional painting".

Certainly the question of to what degree algorithms are tools, as opposed to active collaborators, is a very interesting debate in which the artists can contribute interesting reflections. Although, still this machines are not self-directing agents, we must accept this kind of $\mathrm{Al}$ more of an ideation partner, rather than simply a tool for making new products. 


\section{2. - Machine Learning as a contemporary art practice}

Nowadays artists are beginning to experiment with Artificial Intelligence and change their relationship with technology, which now becomes more of an ideation partner rather than simply a tool for the conception of cultural artifacts.

Memo Akten for example uses emerging technologies as both a medium and a subject matter, looking at its impact on us as individuals, as an extension of our mind and body, and its impact on society, culture, tradition, ritual, etc. On the other side, as seen before, members of french collective Obvious believe that the value of their project lays in the debate it can create through the exposure of new Al tools to the public. From this point of view we can understand Machine Learning as an art practice where an aesthetic sensitivity on the part of the machine might help lead to a friendlier and more sensitive Artificial Intelligence in general.

As maintained by researchers Glenn W. Smith and Frederic Fol Leymarie (2017) we can now begin to think of the machine, not as the artist's subject matter or medium, but as creator or co-creator. With the current development in technology, and with GAN especially, we can without a doubt begin to speak comfortably of the machine as an artist.

According to Blaise Agüera y Arcas (2017) -founder of the Artists and Machine Intelligence program at Google- the transformation of artistic practice and theory that attended the 19th century photographic revolution is equal to the current revolution in machine intelligence, which promises to democratize the means of reproduction and production. Many will remember here an essay of cultural criticism called "The work of art in the age of mechanical reproduction" (1935) by Walter Benjamin, which proposes that the aura of a work of art is devalued by mechanical reproduction. Some may argue -in a clear romantic stance- that modern means of artistic production and reproduction like photography and video destroyed the aesthetic, cultural, and political authority of art. Contrary to this believe we should accept that the diffusion and popular use of different technological innovations, like photography or Machine Learning, will help us explore new domains of art and creativity. Mario Klingemann for example builds art-generating software by feeding photos, video, and line drawings into code borrowed from Machine Learning research. In his opinion he is like a photographer who goes out into the world of Artificial Neural Networks and frames good spots. As Jennifer Sukis Design Principal for AI \& Machine Learning at IBM writes (2018): "We need art to imagine what Al can become, and understand it's impact on who we are becoming". 
Professor Ahmed Elgammal wonders what will happen if we teach machines to generate novel images... Would it generate something that is aesthetically appealing to humans? Would that be considered "art"? The creative artificial system created by Elgammal and his partners at Rutgers University is inspired by the psychological theory of art evolution proposed by Colin Martindale who hypothesized that at any point in time creative artists try to increase the arousal potential of their art to push against habituation. So if we understand scientific research as those investigations conducted for the purpose of contributing towards science by the systematic collection, interpretation and evaluation of data, this new kind of art-based research in Machine Learning will help us imagine what Artificial Intelligence can become and how will influence our technoscientific world.

There is no doubt that in this case Computational Aesthetics help us improve understanding of human aesthetic perception. Because when intelligent machines start generating their own designs and art pieces, free from our aesthetic constraints, how are we as human beings going to be able to understand their own original outcomes? Are we ready to adapt and fall in love with non-antropocentric forms of creativity?

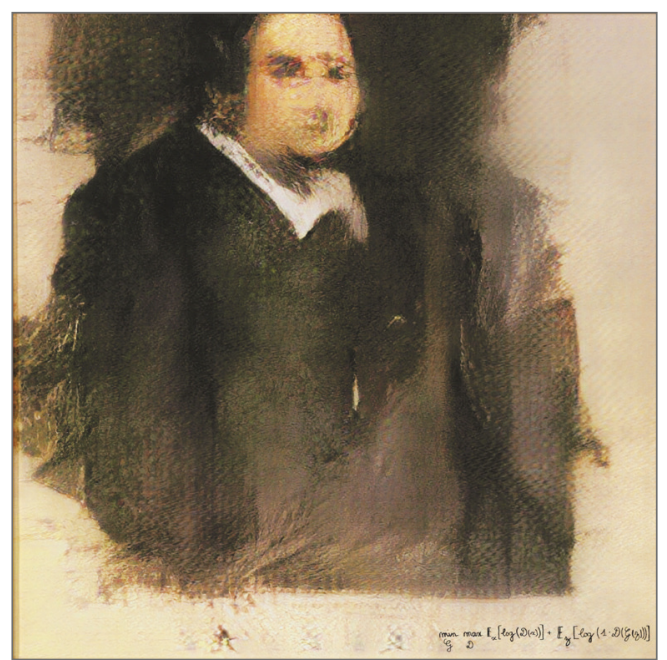

Edmond de Belamy painting. Download here (Public Domain): https://en.wikipedia.org/wiki/Edmond_de_Belamy\#/media/ File:Edmond_de_Belamy.png
Philosopher and art critic Arthur Danto (1998) thought that theory is what takes an artwork up into the world of art and that the role of artistic theories is to make the artworld posible. Danto quoted Leonardo da Vinci by saying ogni dipintore dipinge se ("every painter paints himself"), but today we may want to say ogni automăta dipinge se ("every automata paints himself")... So in order to break through the barrier of disbelief, let's keep moving forward investigating about Synthetic Creative Thinking which might be achieved with the current developments in Cognitive Computing! 


\section{COMPUTATIONAL AESTHETICS AND THE DIGITAL “GESAMTKUNSTWERK”}

\section{1. - Cultural artifacts as a critique of industrialized USE OF AI}

Artisans may have thought that there was no avenue for machines to take over their ethereal reins since something soulless and emotionless does not have the ability for creation and artistic expression: human emotion. But different arts fields (cuisine, fine art, dance, theater, film...) have all made advancements in incorporating machine learning in their crafts.

Fanboys of Frankfurt School of critical theory may argue that modern means of artistic production and reproduction the aesthetic, cultural, and political authority of art. Contrary to this believe we should accept that the diffusion and popular use of different technological innovations, like Machine Learning today, helps us explore new domains of creativity.

When in 2017 Ahmed Elgammal designed a semiautonomous agent called AICAN, this Artificial Intelligence learned existing painting styles and aesthetics and generated new ones of its own. As the researchers explain although the algorithm created appealing images, it lived in an isolated creative space that lacked social context, in contrast with human artists who are inspired by people, places and politics. We humans definitely make art to tell stories and make sense of the world, so because now machines can almost autonomously produce art it doesn't mean they will replace artists. Elgammal also likes to compare this new kind of art made by Artificial Intelligence to photography, a field invented in the early 19th century that wasn't considered art because the popular belief was that a machine was doing much of the work.

In the 20th century since Futurism and Dadaism machines and technologies have been interwoven into the artistic imaginary. According to art historian Andreas Broeckmann (2018) the notion of the machine is predicated on the idea that there is a technical system outside and opposite the human observer, a conceptual space that becomes indefensible once we accept that the prostheses humans carry (devices, drugs they take, food,language...), are not opposite to, but part of the technical formations. 
As Ken Jordan reminds us in his article Defining multimedia (1998), already in 1945 Vannevar Bush's aim was to create a machine that supported the mind's process of free association in the act of creation. By 1950 J.C.R. Licklider in his seminal article Man-computer symbiosis proposed that the computer should act as an extension of the human capabilities for cognition and communication; which included, of course, the manipulation of media.

In a sense when we talk of this human-machine hybridization for the creative process, we are defining what 19th century german composer and theatre director Richard Wagner defined as 'Gesamtkunstwerk'. This term is about how artists have exploited media to create a more total experience and artwork, a term that helps to describe the multisensory immersion in sight and sound we now associate with the advanced technological forms of digital multimedia or what we now call new-media art (the sophisticated technologies that have become available to artists since the late 1980s that can enable the digital production and distribution).

As curator Jasia Reichardt wrote more than 30 years ago, artists have contributed significantly to the current image and meaning of the machine and have celebrated machines since they came into existence, first by depicting them and more recently by using them as tools and assistants. The author questioned: "What will happen when machines make their own art? Will we recognise it and will we accept it?".

And the current deployment of Machine Learning algorithms and Artificial Intelligence techniques that we are seeing in the art world today may be seen as the ultimate 'Gesamtkunstwerk' or total artwork. Because designing artifacts (Al agents) that are not only products or tools, but collaborators or even artistic creators, should be seen as the ultimate frontier in the creative processes. The production and use of technologies as an extension of the human condition has helped us not only to dominate our natural environment, but to know ourselves better as a biological species. As we can see the emerging field of Neural Aesthetics is a promising field that blends art/design and Artificial Neural Networks in order to use the advances in Deep Learning in the artistic field. Not only we need this type of cultural artifacts as a critique of industrialized use of Artificial Intelligence, but also a strict criteria has to be delimited in order to review contemporary art made with Machine Learning techniques... For now, art made with Artificial Intelligence will be a driver of innovation but, exactly as proposed by pioneer computer artist Frieder Nake fifty years ago, not so much a driver of aesthetic change but instead of a critical revolution. 
In this journey this new kind of Computational Aesthetics can help us not only creating synthetic methods that can make applied aesthetic decisions in a similar fashion as humans can, but it can assist us in reevaluating our preconceptions of what artistry is... because, who says that the beholder needs eyes to appreciate beauty? And anyway, isn't art, since the cave paintings in the Stone-Age, a sign of human evolution?

\section{2. - TOWARDS THE CREATION OF AUTONOMOUS ARTI- FICIAL ARTISTS}

Those that argue against the potential for Computational Creativity believe something like that "simulating artistic techniques means also simulating human thinking and reasoning" (...) and may conclude that "this is impossible to do using algorithms or information processing systems". Regardless of this viewpoint, art made by Artificial Intelligence expands the exploration started by Dadaism and Pop-art in territories like authorship, ownership of the work, and whether process is more important than the finished product.

For example Sol Lewitt was experimenting with instruction based artworks in his artwork series called Wall drawings started in 1968, which eventually and until his death, would use algorithms for the design of such creations and teams of assistants for the execution of these pieces. LeWitt neutralised the materiality and took the further step of virtually denying an independent object existence to his art. And it's not coincidence that Al artist Robbie Barrat -who uses Deep Learning and Generative Adversarial Networks in his works- states that his role as a creator is like that of artist Sol Lewitt, best known for writing out instructions or rule sets for creating drawings and then having others execute the rules to create his artwork. Unlike with traditional Generative Art where the artist establishes the code and the computer has no room for interpretation, with this kind of $\mathrm{Al}$ generated artworks the artist is like giving imagination to the machines.

A century ago Marcel Duchamp, pioneer of conceptual art, stated that the artist's idea was the key element in the artistic creation in clear opposition to the classical view that emphasizes a perspective centered around aesthetics. But this new art created by Generative Adversarial Networks radically flips this idea, not just creating a new thought for an object, but apparently creating an artifact capable of doing the 'thinking' and 'painting' for us. And the same way as Robbie Barrat was upset that Obvious used his open-source code to gener- 
ate the much hyped "Edmond de Belamy" painting, it's important to remember that the mentioned code which actually trains and generates the images is from Machine Learning developer Soumith Chintala, and the data to train the agent is accessible online.

"The human is indissolubly linked with imitation: a human being only becomes human at all by imitating other human beings".

(Theodor Adorno)

Ramón López de Mántaras, director of the spanish Artificial Intelligence Research Institute, writes that the fact that we are not conscious of how a creative idea manifests itself does not necessarily imply that a scientific explanation cannot exist (2016). Therefore creativity is not some mystical gift that is beyond scientific study but rather something that can be investigated, simulated, and harnessed for the good of society. Existing computer programs lack too many relevant causal connections to exhibit intentionality while manipulating symbols and learning from that but that doesn't mean that perhaps in a near future a new kind of "embodied" Artificial Intelligences while interacting with their environment may be able to give meaning to their work.

We humans are reluctant to accept that non-anthropomorphic and non-biological agents can be creative because accepting it would have important moral, ethical and social implications. As science journalist Andrea Morris thinks (2018), we have no criteria for recognizing sentience in beings without biological brains and nervous systems. So maybe, Artificial Intelligence -like the Animal Liberation movement decades ago- will question assumptions about the legal and moral state of these intelligent machines, and evidently this new kind of society will therefore have to work on granting rights and acquiring responsibilities towards their respective human, post-human and ecological communities.

As PhD candidates Fabian Offert and Andrey Kurenkov remind us in their fantastic article The past, present, and future of Al art (2019) in Alan Turing's view, and paraphrasing Adorno, we could say that artists follow an intuitive logic, a process like any other rule-bound activity. As pioneer Al artist Harnold Cohen thought when designing his expert system AARON that it "should be possible to devise a set of rules and then, almost without thinking, make the painting by following the rule". This approach is characteristic of a certain type of artist like the classic abstractions of Piet Mondrian from the 1920s and 1930s which were made according to a set of self-imposed regulations: only straight lines were 
allowed, which could meet only at right angles and could be depicted only in a palette of red, blue, and yellow (plus black and white).

Therefore the question if can machines be creative arises from a distorted and romantic notion of creativity, because let's not forget that only during the Renaissance the concepts of freedom and creativity gained momentum until the 19th century when art took its revenge because it started to be considered an act of creativity by the mere fact of being. Later on -at the turn of the 20th century- a transference of the qualities of creativity started towards to the sciences and to nature, concepts which previously were only related to art. Offert and Kurenkow also remind us that contemporary art has not been about image making for a long time, and while most people admire the accomplished craftsmanship of representational artists, works made with Generative Adversarial Networks have the problem that they are always essentially mimetic. While this artifacts will certainly produce interesting variations, a neural network can never distance itself from the world of data it operates on, so they might never produce an image that reflects on the art historical context and shakes our notion of aesthetics.

Already in 1843 Ada Lovelace, now widely considered to be the first programmer, wrote that computers can never be as intelligent as humans because they simply can only do what we program them to do, "only when computers originate things should they be believed to have minds" wrote the british mathematician.

The Lovelace Test, designed in the early 2000 s by a team of computer scientists that developed Watson computer for IBM, looks for genuine autonomous creativity instead of simply manipulating syntax. In Mark Riedl, from Georgia Institute of Technology designed the Lovelace Test 2.0, which is an upgrade from the original version and demands that the Al create art. So we might argue that Deep Learning, as a class of Artificial Intelligence architecture that makes computers learn from experience, might be a candidate to pass the above test because the machine gathers knowledge from the experience of processing different data-sets without the need for a human operator to formally specify all the information. This is a major breakthrough since most of the business and scientific systems developed for the first 50 years of Artificial Intelligence research were all based on rule-based systems inspired in our deductive reasoning, while we as humans usually learn by inductive reasoning which makes broad generalizations from specific observations. 
All of all this gets weirder when artist and a programmer Gene Kogan (2018) talks about the concept of an 'Autonomous Artificial Artist' where he envisions "taking the machine out of machine learning" and putting that total artwork on the Blockchain, a computation model that is the next evolution of a internet. Now that this concept and technology is becoming more common, artists are starting to use it to bring forward a new kind of decentralized organization for creating self-sustaining art. This concept portrays an ecosystem that is able to manage itself autonomously without the need of a centralized power or Artificial Intelligence, only a set of self-executing rules and contracts. From a biological point of view we might compare this to the little cognition of an ant that gets multiplied by the distributed and decentralized social structure of its colony...

\section{CONCLUSION}

Art made with Artificial Neural Networks is being used and advertised as a driver of innovation in the fields of technology development and the art market. But as pioneer computer artist Frieder Nake advanced half a century ago, this kind of novelties are to be used not so much a driver of aesthetic change but instead as a mode of critical revolution.

Unlike with traditional Generative Art where the artist establishes the code and the computer has no room for interpretation, with this kind of artificially generated artworks the artist is like giving imagination to the machines.

What we can for sure learn by creating this Artificial Neural Networks and programming them is how humans by becoming image making artists developed a great sophistication of our creativity which seems to be a glorious accidental product of natural selection... And maybe this way, by reverse engineering our brain, we will someday be in charge of our own evolution. 


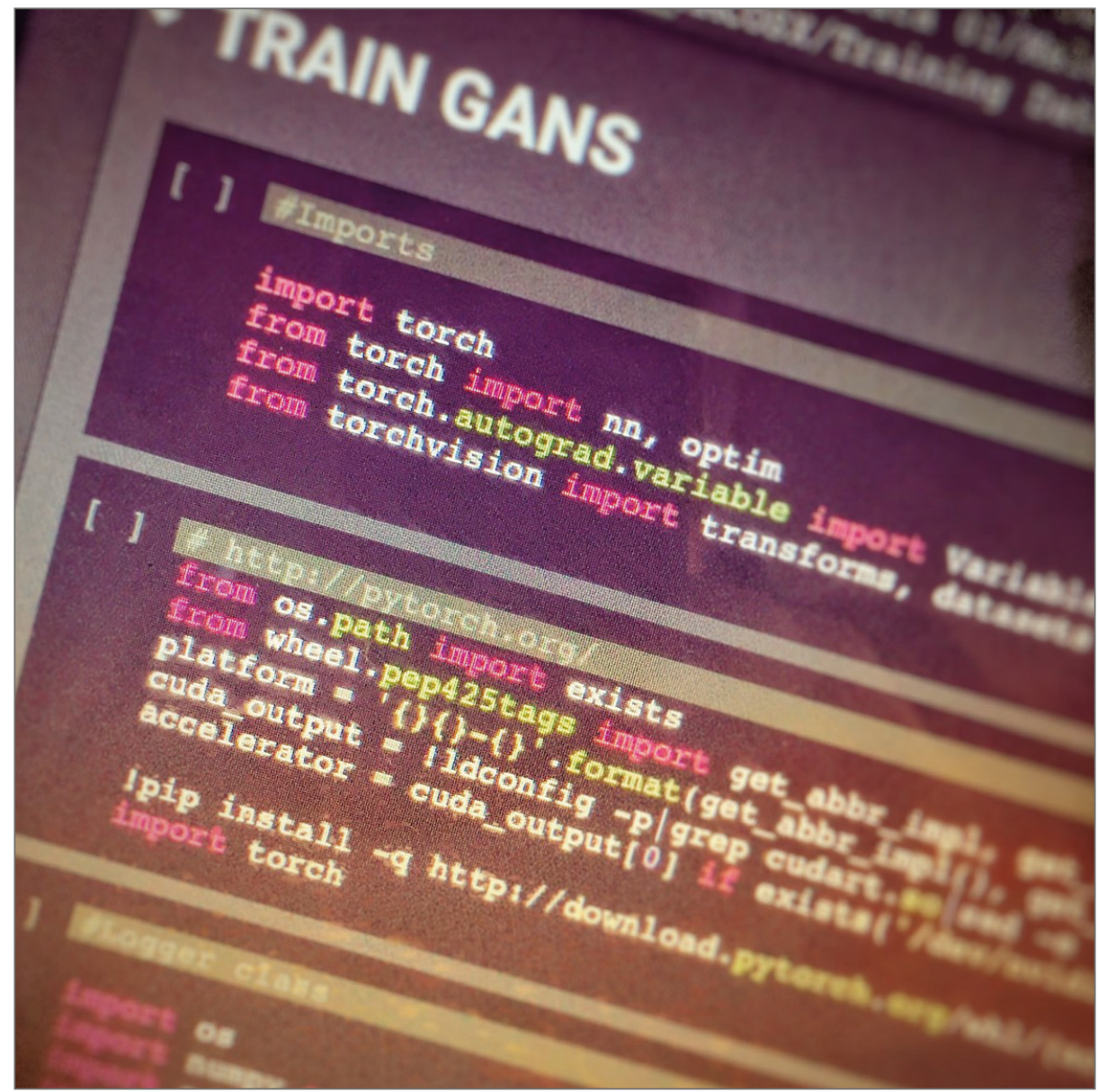

Machine Learning Notebook executing GAN algorithms.

Download here (Copyleft by the author of this article):

https://mikelarbiza.files.wordpress.com/2019/10/train-gans.jpg

\section{References}

Agüera y Arcas, Blaise. 2017. "Art in the age of machine intelligence". Arts 6(4): 29-38. https:// doi.org/10.3390/arts6040018

Akten, Memo. 2018. "Machine learning art: An interview with Memo Akten". Renée Zachariou. Artnome.com, Dec. 16. https://www.artnome.com/news/2018/12/13/machine-learning-artan-interview-with-memo-akten 
Arbiza Goenaga, Mikel. 2018a. "Evolution by design”. MikelArbiza.net (blog), Jul. 31. https:// mikelarbiza.net/2018/07/31/evolution-by-design/

— . 2018b. "Le Witt's immortal paintings". MikelArbiza.net (blog), Jun 18. https://mikelarbiza. net/2018/06/18/le-witts-inmortal-paintings/

— . 2018c. "Machine learning art as scientific research". MikelArbiza.net (blog), Nov. 12. https://mikelarbiza.net/2018/11/12/who-is-edmond-de-bellamy/

— . 2018d. "Robotic citizenship is coming!". MikelArbiza.net (blog), 26 oct. https://mikelarbiza net/2018/10/26/robotic-citizenship-is-coming/

— . 2018e. "The machine will have the idea...". MikelArbiza.net (blog), Jun 18. https://mikelarbiza.net/2018/06/18/le-witts-inmortal-paintings/

— . 2018f. "The true poet is creative". MikelArbiza.net (blog), Feb. 19. https://mikelarbiza. net/2018/02/19/the-true-poet-is-creative/

— . 2018g. "What is blockchain". MikelArbiza.net (blog), April 4. https://mikelarbiza. net/2018/04/04/what-is-blockchain/

— . 2018h. "Who is Edmond de Bellamy?". MikelArbiza.net (blog), Nov. 12. https://mikelarbiza. net/2018/11/12/who-is-edmond-de-bellamy/

— . 2019a. "Artificial Intelligence: a revolution in the art world". MikelArbiza.net (blog), May 7. https://mikelarbiza.net/2019/05/07/artificial-intelligence-a-revolution-in-the-art-world/

— . 2019b. “Computational aesthetics: The beholder needs eyes?". MikelArbiza.net (blog), May 20.https://mikelarbiza.net/2019/05/20/computational-aesthetics-the-beholder-needs-eyes/

Benjamin, Walter. (1935) 2008. The work of art in the age of mechanical reproduction. Translated by J.A. Underwood. London: Penguin

Bhatia, Richa. 2018. "Neural networks do not work like human brains: Let's debunk the myth". Analytics India Magazine, Jan.10. https://analyticsindiamag.com/neural-networks-not-worklike-human-brains-lets-debunk-myth/

Bilal, Afaan. 2018. "Artificial neural networks and deep learning”. Becoming Human, Jan. 31. https://becominghuman.ai/artificial-neural-networks-and-deep-learning-a3c9136f2137

Billitz, Nicole. 2014. "Lovelace 2.0 tests creativity". Versus.com, Nov 24. https://versus.com/en/ news/lovelace-2-0-tests-creativity

Boden, Margaret. 2012. "Creativity \& Al: Asking the right questions; Interview at Oxford University”. By Adam Ford. Youtube video, 26:52, Jan 16. https://youtu.be/5dEXIOiAsaw

Broeckmann, Andreas. 2016. Machine art in the Twentieth Century. Cambridge MA: MIT

Buckner, Cameron \& James Garson. 2019. “Connectionism”. The Stanford Encyclopedia of Philosophy, May 18. https://plato.stanford.edu/archives/fall2019/entries/connectionism/

Çaparlar, Ceyda Özhan \& Aslı Dönmez. 2016. "What is scientific research and how can it be done?". Turkish Journal of Anaesthesiology \& Reanimation 44(4): 212-18

Christie's. 2018. "Is artificial intelligence set to become art's next medium?" Christies.com, Dec. 12 https://www.christies.com/features/A-collaboration-between-two-artists-one-humanone-a-machine-9332-1.aspx

Cohn, Gabe. 2018. “Up for bid, ai art signed 'algorithm”. The New York Times, Oct. 22. https:// www.nytimes.com/2018/10/22/arts/design/christies-art-artificial-intelligence-obvious. html?ref=oembed

Danto, Arthur C. 1998. "The end of art: A philosophical defensa". History and Theory 37(4): 127-43 
Elgammal, Ahmed, Bingchen Liu, Mohamed Elhoseiny\& Marian Mazzone. 2017. "CAN: Creative Adversarial Networks, generating 'art' by learning about styles and deviating from style norms". ArXiv.org, Jun. 21. https://arxiv.org/abs/1706.07068

- Elgammal, Ahmed. 2017. "Generating 'art' by Learning about styles and deviating from style norms". Medium.com, Jun. 26. https://medium.com/@ahmed_elgammal/generatingart-by-learning-about-styles-and-deviating-from-style-norms-8037a13ae027

- Elgammal, Ahmed. 2018. "Meet AICAN, a machine that operates as an autonomous artista". The Conversation, Oct. 17. https://theconversation.com/meet-aican-a-machine-thatoperates-as-an-autonomous-artist-104381

Gayford, Martin. 2016. "Robot art raises questions about human creativity". MIT Technology Review, Feb. 15. https://www.technologyreview.com/2016/02/15/162067/robot-art-raises-questions-about-human-creativity/

Giles, Martin. 2018. “The GANfather: The man who's given machines the gift of imagination”. MIT Technology Review, Feb. 21. https://www.technologyreview.com/2018/02/21/145289/ the-ganfather-the-man-whos-given-machines-the-gift-of-imagination/

Goodfellow, lan J. et al. 2014. "Generative adversarial networks". ArXiv.org, Jun. 10. https:// arxiv.org/abs/1406.2661

Hui, Jonathan. 2018. "GAN: What is generative adversarial networks GAN?". Medium.com, Jun. 19. https://medium.com/@jonathan_hui/gan-whats-generative-adversarial-networks-and-its-application-f39ed278ef09

Invaluable. 2019. "How artificial intelligence is changing the face of art". Invaluable.com, Jan. 29. https://www.invaluable.com/blog/ai-art/

Jordan, Ken. 1998. "Defining multimedia”. IEEE Multimedia 5: 8-15

Knight, Will. 2017. "5 Big predictions for artificial intelligence in 2017". MIT Technology Review, Jan 4. https://www.technologyreview.com/2017/01/04/154761/5-big-predictions-for-artificial-intelligence-in-2017/

Kogan, Gene. 2018. “The neural aesthetic @ ITP-NYU: Lecture 11, Autonomous artificial artists". Youtube video, 2:43:06, Dec. 4. https://www.youtube.com/watch?v=Dqudnjj5wSo

Krome, Sven. 2018. "Decentralized Autonomous Art: Art in the blockchain foreshadows our future". Here 360, Aug. 28. https://360.here.com/decentralized-autonomous-art-art-in-the-blockchain-foreshadows-our-future

Lazic, Sanja. 2017. "Is authorship in art a victim of appropriation?". Widewalls Modern \& Contemporary Art Resource, July 24. https://www.widewalls.ch/authorship-in-art/

Locke, Nancy. 2015. "How photography evolved from science to art". The Conversation, Mar. 11. https://theconversation.com/how-photography-evolved-from-science-to-art-37146

López de Mántaras Badía, Ramón. 2016. "Artificial Intelligence and the arts: Toward computational creativity". In The next step: Exponential life, Jay David Bolter et al.; publishing coordination, Nuria Martínez Deaño; translation, Wade Matthews. Madrid: BBVA

Monoskop. 2020. "Neural aesthetics: A resource on recent work between art/design and artificial neural networks in machine learning". Monoskop.org, May.14. https://monoskop.org/ Neural_aesthetics

Morris, Andrea. 2018. "We need to talk about sentient robots". Forbes.com, Mar. 13. https:// www.forbes.com/sites/andreamorris/2018/03/13/we-need-to-talk-about-sentient-robots/\#2ccae0681b2c 
New Atlas. 2015. "Creative Al: The robots that would be painters". Newatlas.com, Feb. 16. https://newatlas.com/creative-ai-algorithmic-art-painting-fool-aaron/36106/

Offert, Fabian. 2019. "The past, present, and future of Al art". Skynet Today, June 3. https:// www.skynettoday.com/editorials/ai-art-history

Packer, Randall. 2013. "The real history of multimedia". Inside/Out, Sep. 23. https://www.moma org/explore/inside_out/2013/09/23/the-real-history-of-multimedia/

Pearson, Jordan. 2014. "Forget Turing, the Lovelace test has a better shot at spotting Al". Vice. com, Jul. 8. https://www.vice.com/en_us/article/pgaany/forget-turing-the-lovelace-test-hasa-better-shot-at-spotting-ai

Reichardt, Jasia. 1987. "Machines and art". Leonardo 20(4): 367-72

Smith, Glenn W. \& Frederic Fol Leymarie. 2017. "The machine as artist: An introduction". Arts 6(2), 5. https://doi.org/10.3390/arts6020005

Sukis, Jennifer. 2018. "The relationship between art and Al". Medium.com, May 16. https:// medium.com/design-ibm/the-role-of-art-in-ai-31033ad7c54e

Vicent, James. 2018. "How three french students used borrowed code to put the first ai portrait in Christie's". Theverge.com, Oct. 23. https://www.theverge.com/2018/10/23/18013190/ ai-art-portrait-auction-christies-belamy-obvious-robbie-barrat-gans

\section{Notes}

${ }^{1}$ François Chollet. 2017. Twitter communication. Jul. 13. 2017, 02 a.m. https://twitter.com/ fchollet/status/885378870848901120

${ }^{2}$ Robbie Barrat. Twitter communication. Oct. 25 2018, 4:31 a.m. https://twitter.com/videodrome/ status/1055285640420483073

(Artículo recibido: 26-02-20; aceptado: 14-05-20) 